\title{
Influence of magnetic electrodes thicknesses on the transport properties of magnetic tunnel junctions with perpendicular anisotropy
}

Cite as: Appl. Phys. Lett. 105, 052408 (2014); https://doi.org/10.1063/1.4892450

Submitted: 11 March 2014 . Accepted: 26 July 2014 . Published Online: 06 August 2014

Léa Cuchet, Bernard Rodmacq, Stéphane Auffret, Ricardo C. Sousa, and Bernard Dieny
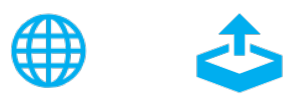

\section{ARTICLES YOU MAY BE INTERESTED IN}

Thick CoFeB with perpendicular magnetic anisotropy in CoFeB-MgO based magnetic tunnel junction

AIP Advances 2, 042182 (2012); https://doi.org/10.1063/1.4771996

Spin torque switching of perpendicular $\mathrm{Ta}|\mathrm{CoFeB}| \mathrm{MgO}-$ based magnetic tunnel junctions Applied Physics Letters 98, 022501 (2011); https://doi.org/10.1063/1.3536482

Perpendicular-anisotropy CoFeB-MgO magnetic tunnel junctions with a MgO/CoFeB/Ta/ $\mathrm{CoFeB} / \mathrm{MgO}$ recording structure

Applied Physics Letters 101, 022414 (2012); https://doi.org/10.1063/1.4736727

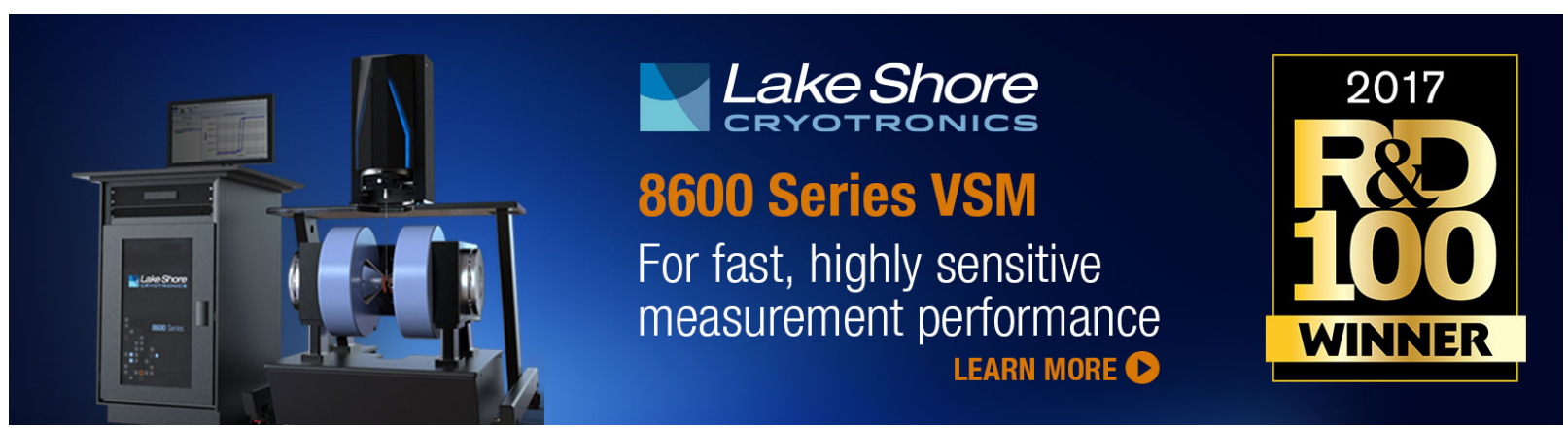




\title{
Influence of magnetic electrodes thicknesses on the transport properties of magnetic tunnel junctions with perpendicular anisotropy
}

\author{
Léa Cuchet, Bernard Rodmacq, Stéphane Auffret, Ricardo C. Sousa, and Bernard Dieny \\ SPINTEC, UMR 8191, CEA-INAC/CNRS/UJF-Grenoble 1/Grenoble-INP, 38054 Grenoble Cedex, France
}

(Received 11 March 2014; accepted 26 July 2014; published online 6 August 2014)

\begin{abstract}
The influence of the bottom and top magnetic electrodes thicknesses on both perpendicular anisotropy and transport properties is studied in $(\mathrm{Co} / \mathrm{Pt}) / \mathrm{Ta} / \mathrm{CoFeB} / \mathrm{MgO} / \mathrm{FeCoB} / \mathrm{Ta}$ magnetic tunnel junctions. By carefully investigating the relative magnetic moment of the two electrodes as a function of their thicknesses, we identify and quantify the presence of magnetically dead layers, likely localized at the interfaces with Ta, that is, $0.33 \mathrm{~nm}$ for the bottom electrode and $0.60 \mathrm{~nm}$ for the top one. Critical thicknesses (spin-reorientation transitions) are determined as 1.60 and $1.65 \mathrm{~nm}$ for bottom and top electrodes, respectively. The tunnel magnetoresistance ratio reaches its maximum value, as soon as both effective (corrected from dead layer) electrode thicknesses exceed 0.6 nm. (C) 2014 AIP Publishing LLC. [http://dx.doi.org/10.1063/1.4892450]
\end{abstract}

Magnetic Random Access Memories (MRAM) based on Magnetic Tunnel Junctions (MTJs) are promising devices as they combine several advantages: non-volatility, high write speed of a few ns, density $\left(\sim 6 \mathrm{~F}^{2}\right)$, infinite endurance $\left(>10^{16}\right.$ write cycles), and radiation hardness. In recent years, research mainly focused on MTJs with perpendicular magnetic anisotropy (PMA), since, compared to their in-plane counterparts, they provide better thermal stability, lower current densities for spin transfer torque (STT) switching for a given memory retention time ${ }^{1}$ and higher storage densities. Standard magnetic junctions are usually composed of a $\mathrm{MgO}$ barrier separating two CoFeB layers, an as-deposited amorphous alloy that has the advantage of giving a large tunnel magnetoresistance (TMR) effect after crystallization in the bcc (100) structure compatible with the texture of the $\mathrm{MgO}$ barrier, leading to strong spin filtering effects. ${ }^{2}$

To introduce PMA in such structures, it is possible to use the properties of $\mathrm{Co} / \mathrm{Pt}$ or $\mathrm{Co} / \mathrm{Pd}$ multilayers. ${ }^{3,4}$ These materials indeed provide large PMA (of the order of $1.2 \mathrm{erg}$ / $\mathrm{cm}^{2}$ for $\mathrm{Co} / \mathrm{Pt}$ interfaces), thanks to their large spin-orbit coupling, but also induce a strong Gilbert damping which is detrimental for spin transfer torque switching. Therefore, using these materials is adequate for the hard reference layer in STT-RAM but not for the soft storage layer. Large perpendicular interfacial anisotropy has been evidenced at metal/oxide interfaces ${ }^{5,6}$ even when the involved materials have weak spin-orbit coupling as in the case of $\mathrm{FeCoB} / \mathrm{MgO}$ interfaces. This allows getting simultaneously large PMA and weak Gilbert damping which is of crucial interest for the storage layer in scalable STT-RAM. This anisotropy at metal/oxide interface has been shown to be very sensitive to the oxygen and boron content along the $\mathrm{MgO}$ interface. ${ }^{7}$ Therefore, the oxidation and annealing conditions, as well as the nature of the layers which control the boron diffusion out of the amorphous $\mathrm{CoFeB}$ layer upon crystallization, play an important role. ${ }^{3,8}$

In this study, we investigate the effect of varying the thicknesses of the bottom and top magnetic electrodes in perpendicular magnetic tunnel junctions, in order to determine their respective critical thicknesses (transition from out-of-plane to in-plane orientation), their magnetically dead layer thicknesses, as well as the influence of magnetic layer thicknesses on their tunnel transport properties.

Samples were deposited by magnetron sputtering, under an Ar pressure of $2 \times 10^{-3}$ millibar. The typical stack of the junctions is the following:

Substrate/Ta3/Pt5/(Co0.5/Pt0.25) $5 / \mathrm{Co} 0.5 / \mathrm{Ru} 0.9 /(\mathrm{Co} 0.5 /$ $\mathrm{Pt} 0.25)_{3} / \mathrm{Co} 0.5 / \mathrm{Ta} 0.3 / \mathrm{CoFeB} / \mathrm{MgO} / \mathrm{FeCoB} / \mathrm{Ta} 1 / \mathrm{Pt} 2$ (all thicknesses given in $\mathrm{nm}$ ), where $\mathrm{CoFeB}$ stands for a Co-rich alloy $\left(\mathrm{Co}_{60} \mathrm{Fe}_{20} \mathrm{~B}_{20}\right)$ while $\mathrm{FeCoB}$ for a Fe-rich one $\left(\mathrm{Fe}_{72} \mathrm{Co}_{8} \mathrm{~B}_{20}\right)$. Substrate denotes $\mathrm{Si} / \mathrm{SiO}_{2} 50 / \mathrm{Ta} 3 / \mathrm{CuN} 60$, in order to allow transport measurements. The bottom hard electrode is a synthetic $(\mathrm{Co} / \mathrm{Pt})$ antiferromagnet $(\mathrm{SAF})$ coupled to the $\mathrm{CoFeB}$ layer through a thin $(0.3 \mathrm{~nm})$ Ta spacer. This thin nanocrystallized Ta layer allows a structural transition between the $\mathrm{Pt} / \mathrm{Co}$ upper part of the SAF (3-fold symmetry fcc structure) and the $\mathrm{CoFeB}$ layer (4-fold symmetry bcc structure in contact with the $\mathrm{MgO}$ barrier) in order to get a high TMR signal, still keeping a strong magnetic coupling between both $\mathrm{Co}$ and CoFeB layers. ${ }^{9,10}$ The use of $\mathrm{Co} / \mathrm{Pt}$ multilayers allows getting strong perpendicular anisotropy, due to their interfacial anisotropy properties. ${ }^{11}$

The storage layer is the top $\mathrm{FeCoB}$ electrode, and the $\mathrm{MgO}$ tunnel barrier is obtained by natural oxidation of a $1.4 \mathrm{~nm}$ thick $\mathrm{Mg}$ metallic layer. All deposition rates are of the order of $0.05 \mathrm{~nm} / \mathrm{s}$, leading to a negligible uncertainty on the $\mathrm{CoFeB}$ and $\mathrm{FeCoB}$ thicknesses (deposition times for individual layers varying between about 10 to $30 \mathrm{~s}$, depending on their thickness). The $\mathrm{CoFeB}$ and $\mathrm{FeCoB}$ thicknesses are varied independently (the bottom $\mathrm{CoFeB}$ thickness is kept at $1.2 \mathrm{~nm}$ while the top $\mathrm{FeCoB}$ thickness varies between 0.8 and $1.8 \mathrm{~nm}$, and the top $\mathrm{FeCoB}$ thickness is kept at $1.5 \mathrm{~nm}$ while the bottom $\mathrm{CoFeB}$ thickness varies between 0.5 and $2.0 \mathrm{~nm})$. Samples were then vacuum-annealed $\left(10^{-6}\right.$ millibar) for $1 \mathrm{~h}$ at $300^{\circ} \mathrm{C}$ with no external magnetic field.

From the magnetic cycles measured by Vibrating Sample Magnetometry (VSM), a technique which cannot in general give absolute magnetization values, we extract the relative contributions of the different magnetic layers per unit area. Denoting them by $\mathrm{M}_{1}, \mathrm{M}_{2}$, and $\mathrm{M}_{3}\left(\mathrm{M}_{1} / \mathrm{Ru} / \mathrm{M}_{2} /\right.$ 
$\mathrm{MgO} / \mathrm{M}_{3}$ ), there are always two constant contributions when varying the $\mathrm{CoFeB}$ or $\mathrm{FeCoB}$ thicknesses, and these constant contributions can be used for normalization.

To relate magnetic to transport properties in our junctions, the Current In-Plane Tunneling technique (CIPT) is used, which allows measuring tunnel magnetoresistance on full-sheet samples without any patterning. ${ }^{12}$ For such measurements, the resistances of the bottom and top electrodes must be adapted to the RA (resistance-area) product of the $\mathrm{MgO}$ barrier, of the order of $30 \Omega \mu \mathrm{m}^{2}$ in our case. Samples are thus deposited on a $60 \mathrm{~nm}$ thick CuN buffer layer and covered by a $30 \mathrm{~nm}$ thick Ru layer.

Magnetic cycles measured with a perpendicular field by VSM for varying $\mathrm{CoFeB}$ and $\mathrm{FeCoB}$ thicknesses are presented in Figure 1. Coming from positive field saturation, the high-field transition corresponds to the magnetization reversal of the top $\mathrm{PtCo} / \mathrm{CoFeB}$ part of the SAF hard electrode, that is to say M2. This can be inferred from the increase of its relative contribution as the bottom $\mathrm{CoFeB}$ thickness increases (left curves). Indeed, in the normalized cycles presented in Figure 1, the amplitude of the signal at zero field keeps decreasing as the $\mathrm{CoFeB}$ thickness increases. Besides, this is also confirmed by transport measurements, since the high-resistance state is measured for positive applied fields, indicating that the bottom $\mathrm{CoFeB}$ electrode is antiparallel to the top $\mathrm{FeCoB}$ soft layer. The central transition corresponds to the reversal of the top $\mathrm{FeCoB}$ free layer (M3), and the last transition, corresponding to the largest coercive field, represents the hardest part of the SAF M1. The magnetic coupling through the $\mathrm{MgO}$ barrier is antiparallel, as usually observed in perpendicular junctions, ${ }^{10,13}$ and amounts here to about $-50 \mathrm{Oe}$, without any significant or systematic variation as a function of electrode thicknesses. This antiferromagnetic coupling manifests itself by a shift towards negative fields of the minor loop performed on the free layer (central part of the magnetic cycles, not shown in Figure 1) since, still coming from positive fields, the magnetic layer M2 on the other side of the $\mathrm{MgO}$ barrier is already in the "down" direction. M3 thus tends to stay in the "up" direction even if the applied magnetic field turns negative.

For bottom $\mathrm{CoFeB}$ thicknesses of $0.8,1.0$, or $1.2 \mathrm{~nm}$, a perfectly horizontal magnetic signal is measured between sharp transitions, reflecting that the anisotropy is perpendicular in the whole structure (Figure 1, left). In contrast, a magnetically hard signal appears for a thickness of $1.6 \mathrm{~nm}$. This signal is not symmetrical with respect to the applied field, which means that it originates from the SAF electrode. This indicates that the magnetization of the upper part of the SAF (M2) starts to tilt away from the perpendicular direction. Decreasing the applied field thus progressively stabilizes M2
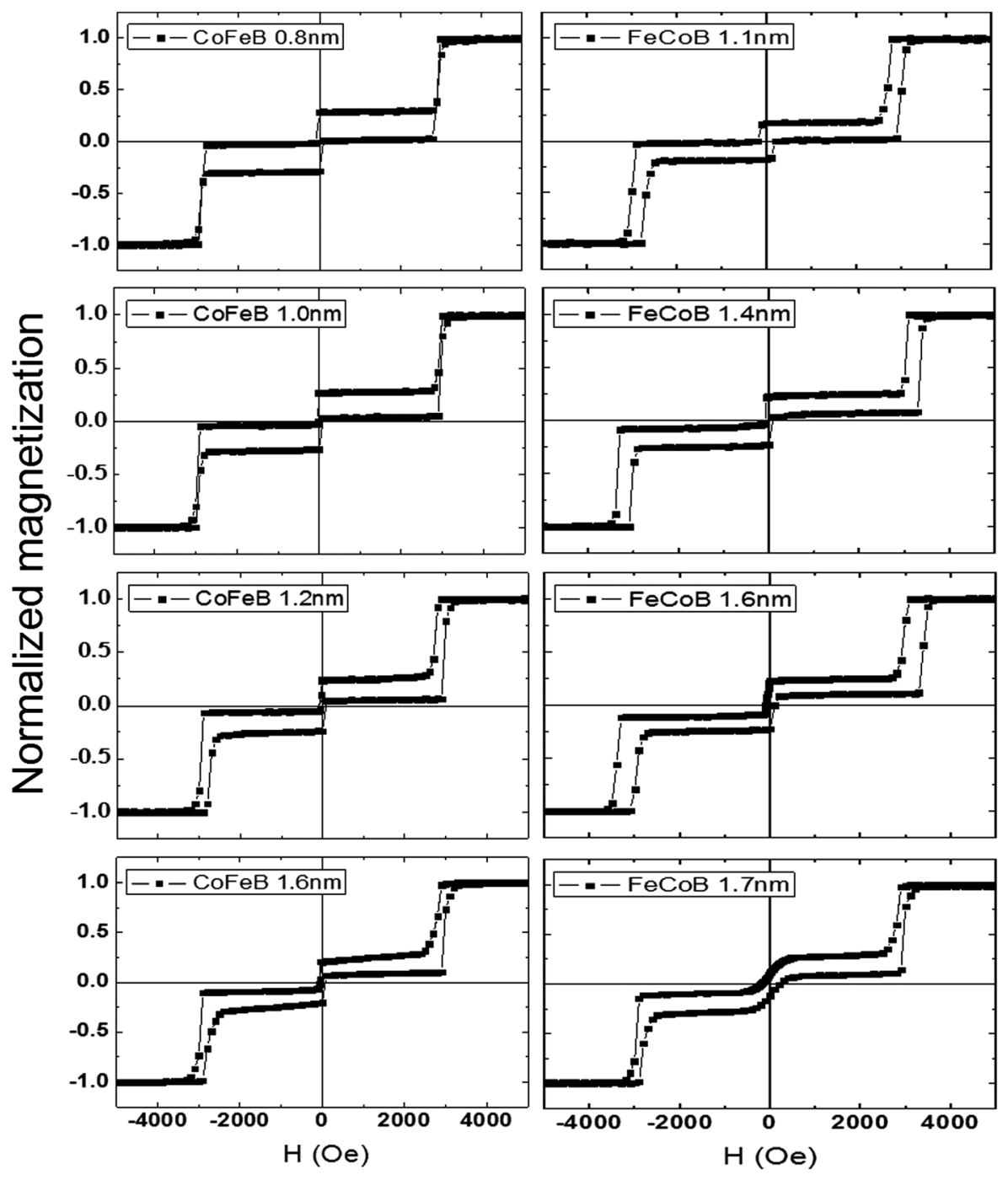

FIG. 1. Magnetic cycles measured by VSM with a perpendicular field for varying thicknesses of bottom $\mathrm{CoFeB}$ (left) and top $\mathrm{FeCoB}$ (right) electrodes, keeping thicknesses of $1.5 \mathrm{~nm}$ for the top $\mathrm{FeCoB}$ layer thickness and $1.2 \mathrm{~nm}$ for the bottom $\mathrm{CoFeB}$ layer one, respectively. 
in the perpendicular negative direction, explaining why no slope is observed in negative applied field. When the thickness of the top $\mathrm{FeCoB}$ layer increases (Figure 1, right), the easy magnetization axis of the soft layer goes from perpendicular-to-plane to in-plane, a characteristic hard-axis behavior being visible for a thickness of $1.7 \mathrm{~nm}$. Although it is not possible to accurately determine the critical thickness (out-of-plane to in-plane reorientation) of the bottom $\mathrm{CoFeB}$ electrode (of the order of $1.6 \mathrm{~nm}$ ), the measurement of the variation of the anisotropy field with the thickness of the top $\mathrm{FeCoB}$ electrode in the vicinity of the reorientation transition leads to a critical thickness of $1.65 \mathrm{~nm}$.

Reminding that our structures can be schematically represented as $\mathrm{M}_{1} / \mathrm{Ru} / \mathrm{M}_{2} / \mathrm{MgO} / \mathrm{M}_{3}$, we present in Figure 2 the variations of $\mathrm{M}_{2} / \mathrm{M}_{1}$ as a function of the bottom $\mathrm{CoFeB}$ thickness and $\mathrm{M}_{3} / \mathrm{M}_{1}$ as a function of the top $\mathrm{FeCoB}$ thickness.

The $\mathrm{M}_{2} / \mathrm{M}_{1}$ ratio can be expressed as

$$
\left.\mathrm{M}_{2} / \mathrm{M}_{1}=\left(4 \mathrm{t}_{\mathrm{Co}} \mathrm{Ms}_{\mathrm{Co}}+\left(\mathrm{t}_{\mathrm{CoFeB}}-\mathrm{t}_{\mathrm{d}}\right) \mathrm{Ms}_{\mathrm{CoFeB}}\right)\right) /\left(6 \mathrm{t}_{\mathrm{Co}} \mathrm{Ms}_{\mathrm{Co}}\right) \text {, }
$$

$t_{\text {Co }}$ being the thickness of the Co layers in the Pt/Co multilayers, $t_{d}$ the magnetic dead layer thickness of the $\mathrm{CoFeB}$ layer, and Ms the saturation magnetizations of the Co or $\mathrm{CoFeB}$ layers. From Figure 2(a), we extract a magnetically dead layer thickness $t_{d}$ of $0.33 \pm 0.02 \mathrm{~nm}$ for the bottom $\mathrm{CoFeB}$ layer, which corresponds to the intercept between the linear fit and the theoretical ratio $\mathrm{M}_{2} / \mathrm{M}_{1}$ without any $\mathrm{CoFeB}$ contribution, that is, $2 / 3$. This treatment assumes that all ten individual Co layers in both bottom and top $(\mathrm{Co} / \mathrm{Pt})$ multilayers have the same saturation magnetizations, despite different interfaces for a few of them $(\mathrm{Co} / \mathrm{Ru}, \mathrm{Ru} / \mathrm{Co}$, and $\mathrm{Co} / \mathrm{Ta}$ ).

This magnetically dead layer of $0.33 \mathrm{~nm}$ is probably located at the bottom interface with the Ta insertion layer. Knowing that $\mathrm{M}_{1}$ is composed of 6 Co layers $0.5 \mathrm{~nm}$ thick each, with a saturation magnetization $\mathrm{Ms}_{\mathrm{Co}}=1200 \mathrm{emu} . \mathrm{cm}^{-3},{ }^{14}$ that is, $15 \%$ smaller than the bulk Co value due to Co-Pt interdiffusion, the fitted slope gives a saturation magnetization of the bottom $\mathrm{CoFeB}$ layer $\mathrm{Ms}_{\mathrm{CoFeB}}$ of $600 \pm 30 \mathrm{emu}^{\mathrm{cm}}{ }^{-3}$. One can note that a similar magnetically dead layer thickness is obtained for junctions where the bottom magnetic layer is made of an Fe-rich $\mathrm{FeCoB}$ alloy instead of a Co-rich $\mathrm{CoFeB}$ one (curves not shown), indicating that it does not depend on the $\mathrm{Co} / \mathrm{Fe}$ atomic ratio of the electrode.
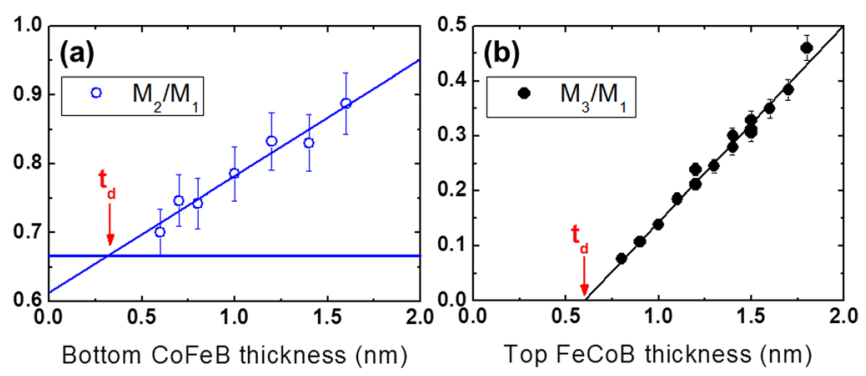

FIG. 2. Magnetization ratio as a function of the bottom $\mathrm{CoFeB}$ (a) and top $\mathrm{FeCoB}(\mathrm{b})$ thicknesses. The horizontal line in the left figure corresponds to the magnetization ratio $M_{2} / M_{1}$ for zero CoFeB thickness, that is to say, $2 / 3$. Error bars correspond to a $5 \%$ uncertainty on the VSM measurements.
A similar data treatment for the top $\mathrm{FeCoB}$ layer can be performed, using this time the $M_{3} / M_{1}$ ratio

$$
\mathrm{M}_{3} / \mathrm{M}_{1}=\left(\mathrm{t}_{\mathrm{FeCoB}}-\mathrm{t}_{\mathrm{d}}\right) \mathrm{Ms}_{\mathrm{FeCoB}} /\left(6 \mathrm{t}_{\mathrm{Co}} \mathrm{Ms}_{\mathrm{Co}}\right) .
$$

In this case (Figure 2(b)), the magnetically dead layer thickness $t_{d}$ of the upper $\mathrm{FeCoB}$ electrode amounts to $0.60 \pm 0.04 \mathrm{~nm}$, with a saturation magnetization $\mathrm{Ms}_{\mathrm{FeCoB}}$ of $1300 \pm 60 \mathrm{emu}^{\mathrm{cm}} \mathrm{cm}^{-3}$. This magnetically dead layer thickness, probably now located at the top $\mathrm{FeCoB} / \mathrm{Ta}$ interface, is larger than the one obtained for the bottom $\mathrm{CoFeB}$ electrode, and may result from the thicker $(1.0 \mathrm{~nm})$ Ta capping layer, compared to the $0.3 \mathrm{~nm}$ Ta insertion layer on which the bottom $\mathrm{CoFeB}$ layer grows. For both Fe-rich and Co-rich electrodes, saturation magnetizations qualitatively agree with those quoted in the literature, ${ }^{3,7}$ which however show a rather large scatter. Such a scatter can be attributed to the strong dependence of saturation magnetization on annealing conditions ${ }^{15}$ resulting from varying residual boron concentrations.

In order to check the validity of our treatment, we present in Figure 3 the variation of the ratio $M_{3} / M_{1}$ (for varying $\mathrm{M}_{2}$ ) and $\mathrm{M}_{2} / \mathrm{M}_{1}$ (for varying $\mathrm{M}_{3}$ ) as a function of bottom and top electrodes thicknesses, respectively. These ratios are found reasonably constant and equal to the theoretical ones $(0.32 \pm 0.03)$ for $\mathrm{M}_{3} / \mathrm{M}_{1}$ and $0.81 \pm 0.08$ for $\left.\mathrm{M}_{2} / \mathrm{M}_{1}\right)$. These expected values are calculated using the saturation magnetizations and the bottom and top magnetically dead layers mentioned above.

We do not try in this Letter to extract from the present measurements values of interface and volume anisotropy energies, as is usually done in the recent literature, ${ }^{3,9,15-19}$ by plotting the effective anisotropy energy $\left(\mathrm{K}_{\mathrm{eff}}=\mathrm{H}_{\mathrm{an}} \mathrm{Ms} / 2\right.$, where $\mathrm{H}_{\mathrm{an}}$ is the anisotropy field and Ms the saturation magnetization) times magnetic thickness as a function of magnetic thickness, and extracting volume and interface terms from the slope and zero intercept of such an ideally linear plot. ${ }^{11}$ The reason is that anisotropy fields of both bottom and top electrodes are not different enough (especially for small thicknesses) to be determined independently in such junctions. A solution would be to study single electrodes, or junctions in which one of the bottom or top electrode is rendered non-magnetic, by using $\mathrm{CoFeB}$ or $\mathrm{FeCoB}$ layers on the

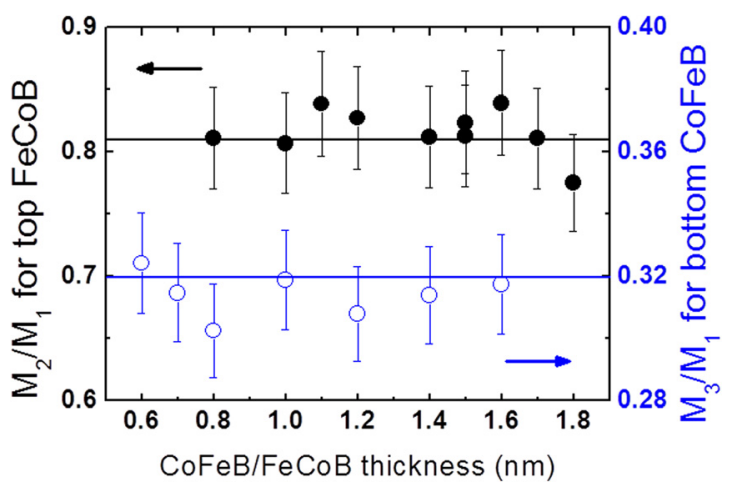

FIG. 3. Magnetization ratio of the constant magnetic contributions M2/M1 as a function of top $\mathrm{FeCoB}$ thickness, filled black circles, left-hand scale, and M3/M1 as a function of bottom CoFeB thickness, open blue circles, right-hand scale. Horizontal lines correspond to the theoretical values, 0.81 and 0.32 , respectively. 

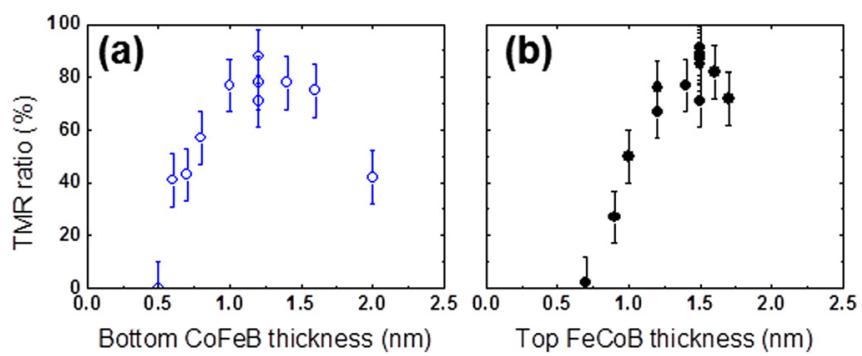

FIG. 4. TMR ratio as a function of bottom $\mathrm{CoFeB}$ (a) and top $\mathrm{FeCoB}$ (b) thicknesses.

other side of the $\mathrm{MgO}$ barrier with a thickness smaller than their dead layer ones. One should assume in this case that such modifications do not have any impact on the growthinduced properties of the rest of the structure. However, we often observed different anisotropy properties in single electrodes, compared to junctions in which one of the magnetic electrodes is rendered non-magnetic, or to full junctions. An explanation could be that, since boron atoms migrate out of the CoFeB layer upon annealing, varying the magnetic thickness could modify the amount of boron atoms at the oxide and cap (seed) interfaces, ${ }^{7}$ having consequences on their anisotropy properties.

We now correlate magnetic and transport properties by measuring the tunnel magnetoresistance of full-sheet samples with the Current In-Plane Tunneling (CIPT) technique. Figure 4(a) shows that, as the thickness of the bottom CoFeB electrode increases, the TMR ratio increases to about $90 \%$ for thicknesses between 1.0 and $1.6 \mathrm{~nm}$, followed by a decrease when a low-field slope appears in the $\mathrm{M}(\mathrm{H})$ curves. This slope is attributed to a progressive tilt of the magnetization of the upper part of the SAF (denoted as $\mathrm{M}_{2}$ ) away from the perpendicular direction, as indicated by the magnetic cycles of Figure 1 (left). Perfect parallel and antiparallel states can, thus, not be reached anymore, leading to an artificial decrease of the TMR ratio. Similarly, samples with varying top FeCoB thickness (Figure 4(b)) show an increase of TMR with $\mathrm{FeCoB}$ thickness, with an asymptotic value between 1.2 and $1.7 \mathrm{~nm}$. The maximum TMR value is not obtained at the same thickness for both electrodes, due to the fact that their respective dead layers thicknesses differ.

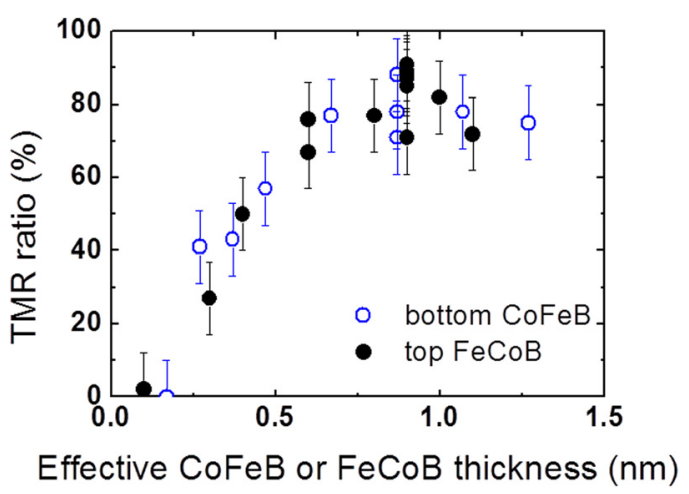

FIG. 5. TMR ratio as a function of bottom $\mathrm{CoFeB}$ (blue open circles) and top FeCoB (black filled circles) thicknesses, respectively, after substraction of their corresponding dead layer thicknesses.
A plot of the TMR ratio as a function of the effective magnetic thickness, i.e., correcting the nominal values from their dead layers contributions, gives identical thickness dependences, as can be seen on Figure 5. The TMR ratio starts increasing as soon as the effective magnetic thickness reaches $0.3 \mathrm{~nm}$. One can also note that the measured RA product of our junctions is essentially constant at about $30 \Omega$ $\mu \mathrm{m}^{2}$ on both $\mathrm{CoFeB}$ and $\mathrm{FeCoB}$ thickness ranges, showing that the TMR decrease for small magnetic thicknesses is not linked to some deterioration of the quality of the $\mathrm{MgO}$ barrier, but rather to a progressive decrease of the electron polarization through thinner magnetic electrodes.

Finally, one can note that the maximum TMR value $(90 \%)$ obtained in these perpendicular junctions prepared in our sputtering tool is slightly lower than the one obtained in corresponding in-plane top-pinned junctions, with a TMR of the order of $120 \%$ (unpublished). This difference can result from a deterioration of the quality of the $\mathrm{MgO}$ barrier due to the $(\mathrm{Co} / \mathrm{Pt})$ multilayer SAF structure on which it grows, possibly leading to some increased roughness of the barrier. This could be related to the slightly larger RA product we measure on perpendicular junctions compared to in-plane ones, which give a RA product around $20 \Omega \mu \mathrm{m}^{2}$.

In conclusion, the influence of magnetic thicknesses of bottom $\mathrm{CoFeB}$ and top $\mathrm{FeCoB}$ electrodes of perpendicular tunnel junctions on their tunnel transport properties was studied. Quantitative analysis of relative magnetization measurements indicates that dead layers exist in the bottom and top magnetic electrodes, probably located at the bottom $\mathrm{Ta} / \mathrm{CoFeB}$ and top $\mathrm{FeCoB} / \mathrm{Ta}$ interfaces, with respective thicknesses of $0.33 \mathrm{~nm}$ for the bottom electrode and $0.60 \mathrm{~nm}$ for the top one. Critical thicknesses, i.e., transitions from out-of-plane to in-plane orientation of the magnetization, are found equal to $1.60 \mathrm{~nm}$ for the bottom $\mathrm{CoFeB}$ layer and $1.65 \mathrm{~nm}$ for the top $\mathrm{FeCoB}$ one. In the thickness range where both top and bottom magnetizations are out-of-plane, or can be brought out-of-plane thanks to the applied field, we observe a similar thickness dependence on effective magnetic thickness (i.e., corrected from magnetically dead layer thickness) of the TMR ratio for both electrodes. For both bottom and top magnetic thicknesses, the TMR ratio saturates to a value of about $90 \%$ as soon as both effective magnetic thicknesses reach $0.6 \mathrm{~nm}$.

This work benefited from financial support of the ANR (French National Research Agency) under project ANRNANO PATHOS and of the European Union under ERC HYMAGINE Project No. 246942. CIPT measurements were carried out at Crocus Technology.

${ }^{1}$ O. G. Heinonen and D. V. Dimitrov, J. Appl. Phys. 108, 014305 (2010).

${ }^{2}$ D. D. Djayaprawira, K. Tsunekawa, M. Nagai, H. Maehara, S. Yagamata, N. Watanabe, S. Yuasa, and K. Ando, Appl. Phys. Lett. 86, 092502 (2005).

${ }^{3}$ D. C. Worledge, G. Hu, D. W. Abraham, J. Z. Sun, P. L. Trouilloud, J. Nowak, S. Brown, M. C. Gaidis, E. J. O'Sullivan, and R. P. Robertazzi, Appl. Phys. Lett. 98, 022501 (2011).

${ }^{4}$ K. Mizunuma, M. Yamanouchi, S. Ikeda, H. Sato, H. Yamamoto, H.-D. Gan, K. Miura, J. Hayakawa, F. Matsukura, and H. Ohno, Appl. Phys. Express 4, 023002 (2011).

${ }^{5}$ S. Monso, B. Rodmacq, S. Auffret, G. Casali, F. Fettar, B. Gilles, B. Dieny, and P. Boyer, Appl. Phys. Lett. 80, 4157-4159 (2002). 
${ }^{6}$ B. Rodmacq, A. Manchon, C. Ducruet, S. Auffret, and B. Dieny, Phys. Rev. B 79, 024423 (2009).

${ }^{7}$ S. Ikeda, R. Koizumi, H. Sato, M. Yamanouchi, K. Miura, K. Mizunuma, H.-D. Gan, F. Matsukura, and H. Ohno, IEEE Trans. Magn. 48, 3829-3832 (2012).

${ }^{8}$ H. Yamamoto, J. Hayakawa, K. Miura, K. Ito, H. Matsuoka, S. Ikeda, and H. Ohno, Appl. Phys. Express 5, 053002 (2012).

${ }^{9}$ V. Sokalski, M. T. Moneck, E. Yang, and J.-G. Zhu, Appl. Phys. Lett. 101, 072411 (2012).

${ }^{10}$ L. Cuchet, B. Rodmacq, S. Auffret, R. C. Sousa, C. Ducruet, and B. Dieny, Appl. Phys. Lett. 103, 052402 (2013).

${ }^{11}$ M. T. Johnson, P. J. H. Bloemen, F. J. A. den Broeder, and J. J. de Vries, Rep. Prog. Phys. 59, 1409-1458 (1996).

${ }^{12}$ D. C. Worledge and P. L. Trouilloud, Appl. Phys. Lett. 83, 84-86 (2003).
${ }^{13}$ L. E. Nistor, B. Rodmacq, S. Auffret, A. Schuhl, M. Chshiev, and B. Dieny, Phys. Rev. B 81, 220407 (2010).

${ }^{14}$ S. Bandiera, R. C. Sousa, Y. Dahmane, C. Ducruet, C. Portemont, V. Baltz, S. Auffret, I. L. Prejbeanu, and B. Dieny, IEEE Magn. Lett. 1, 3000204 (2010).

${ }^{15}$ A. Natarajarathinam, Z. R. Tadisina, T. Mewes, S. Watts, E. Chen, and S. Gupta, J. Appl. Phys. 112, 053909 (2012).

${ }^{16}$ T. Liu, J. W. Cai, and Li Sun, AIP Adv. 2, 032151 (2012).

${ }^{17}$ V. B. Naik, H. Meng, and R. Sbiaa, AIP Adv. 2, 042182 (2012).

${ }^{18}$ J. Sinha, M. Hayashi, A. J. Kellock, S. Fukami, M. Yamanouchi, H. Sato, S. Ikeda, S. Mitani, S.-H. Yang, S. S. P. Parkin, and H. Ohno, Appl. Phys. Lett. 102, 242405 (2013).

${ }^{19}$ V. Sokalski, D. M. Bromberg, M. T. Moneck, E. Yang, and J.-G. Zhu, IEEE Trans. Magn. 49, 4383-4385 (2013). 